\title{
Phylogenomic analysis of cytochrome P450 multigene family and their differential expression analysis in Solanum lycopersicum L. suggested tissue specific promoters
}

\author{
A. P. Vasav and V. T. Barvkar*
}

\begin{abstract}
Background: Cytochrome P450 (P450) is a functionally diverse and multifamily class of enzymes which catalyses vast variety of biochemical reactions. P450 genes play regulatory role in growth, development and secondary metabolite biosynthesis. Solanum lycopersicum L. (Tomato) is an economically important crop plant and model system for various studies with massive genomic data. The comprehensive identification and characterization of P450 genes was lacking. Probing tomato genome for P450 identification would provide valuable information about the functions and evolution of the P450 gene family.

Results: In the present study, we have identified 233 P450 genes from tomato genome along with conserved motifs. Through the phylogenetic analysis of Solanum lycopersicum P450 (SIP450) protein sequences, they were classified into two major clades and nine clans further divided into 42 families. RT-qPCR analysis of selected six candidate genes were corroborated with digital expression profile. Out of 233 S/P450 genes, 73 showed expression evidence in 19 tissues of tomato. Out of 22 intron gain/loss positions, two positions were conserved in tomato P450 genes supporting intron late theory of intron evolution in SIP450 families. The comparison between tomato and other related plant P450s families showed that CYP728, CYP733, CYP80, CYP92, CYP736 and CYP749 families have been evolved in tomato and few higher plants whereas lost from Arabidopsis. The global promoter analysis of SIP450 against all the protein coding genes, coupled with expression data, revealed statistical overrepresentation of few promoter motifs in SIP450 genes which were highly expressed in specific tissue of tomato. Hence, these identified promoter motifs can be pursued further as tissue specific promoter that are driving expression of respective SIP450.
\end{abstract}

Conclusions: The phylogenetic analysis and expression profiles of tomato P450 gene family offers essential genomic resource for their functional characterization. This study allows comparison of S/P450 gene family with other Solanaceae members which are also economically important and attempt to classify functionally important SIP450 genes into groups and families. This report would enable researchers working on Tomato P450 to select appropriate candidate genes from huge repertoire of P450 genes depending on their phylogenetic class, tissue specific expression and promoter prevalence.

Keywords: Cytochrome P450, Phylogeny, Intron map, Genome-wide promoter analysis, Tissue specific promoter

\footnotetext{
*Correspondence: bvitthal@unipune.ac.in; vbarvkar@gmail.com

Department of Botany, Savitribai Phule Pune University, Pune 411007, India

(c) The Author(s). 2019 Open Access This article is distributed under the terms of the Creative Commons Attribution 4.0 International License (http://creativecommons.org/licenses/by/4.0/), which permits unrestricted use, distribution, and reproduction in any medium, provided you give appropriate credit to the original author(s) and the source, provide a link to the Creative Commons license, and indicate if changes were made. The Creative Commons Public Domain Dedication waiver (http://creativecommons.org/publicdomain/zero/1.0/) applies to the data made available in this article, unless otherwise stated.
} 


\section{Background}

Cytochrome P450 (P450) belongs to a very divergent multigene family present in all living organisms. In angiosperms, approximately 300 genes are speculated per genome in 50 plant families [1]. The P450 monooxygenases are heme-thiolate enzymes, which catalyse broad range of chemical reactions like epoxidation, sulfoxidation, dehalogenation, dealkylation, $\mathrm{C}-\mathrm{C}$ cleavage, ring extension, and reduction with the help of oxygen and NADPH [2]. They are involved in the oxidative metabolism of various endogenous and exogenous compounds like herbicides, pesticides and xenobiotics [3, 4]. The P450 proteins, present in plants are membrane bound and difficult to characterize [5]. The molecular mass of P450 from plant origin ranges from 45 to $62 \mathrm{kDa}$ with an average molecular mass of $55 \mathrm{kDa}$. They possess four conserved key domains namely heme binding domain, I-helix, K-helix and PERF/W domain [6]. Heme-binding signature motif has 10 conserved residues among which cysteine is highly conserved. This heme-iron motif has a binding site for oxygen and various compounds involved in drug metabolism [7]. The P450 gene family is third largest gene family present in Arabidopsis. Most of the P450 studied in plants are localized in the endoplasmic reticulum, chloroplast or mitochondria and other secretary pathways [8]. They are involved in many biosynthetic pathways such as alkaloids, flavonoids, lignans, isoprenoids, phenolics, antioxidants and phenylpropanoid [8-10]. The P450 genes are crucial in metabolism and tolerance to allelochemicals in plants as well as in animals [11]. The gene families CYP90, CYP724 and CYP734 are involved in biosynthesis of steroidal saponins and glycoalkaloids. Different types of glycoalkaloids present in all members of Solanaceae family are vital compounds but toxic to other living organisms [12]. The P450 proteins are involved in the biosynthesis of aglycones from cholesterol by oxygenation, transamination and cyclization at different carbon positions. The P450 mediated derivatization of glycoalkaloids made them less toxic and during course of domestication solanaceae members with less amount of toxic glycoalkaloids have been selected [13].

Availability of whole genome sequences of large number of plant species allowed the genome wide identification of P450 multigene family in different plant species, namely soybean (Glycin max) [14], mulberry (Morus notabilis) [15], flax (Linum usitatissimum) [16] and tobacco (Nicotiana tabacum) [17]. The draft genome sequence of tomato (Solanum lycopersicum) was made publicly available in 2012 which provides an opportunity for genome-wide study of tomato specific gene families [18]. Tomato (Solanum lycopersicum L.) is an economically important crop and routinely used model plant for fruit ripening, plant-pathogen interaction and molecular genetics mapping [19]. However, very few P450 genes have been reported and functionally annotated from Tomato. Moreover, no comprehensive genome-wide study of these genes has been reported until date. Therefore. in this study, we attempt to classify functionally important P450 genes into groups and families according to standard P450 nomenclature committee [20, 21]. Understanding the molecular evolution, differential expression in different tissue types as well as intron and promoter analysis of SlP450 genes will pave the way for functional characterization of important candidate genes.

\section{Methods}

Identification of P450 genes from the tomato genome

The Arabidopsis thaliana P450 genes were downloaded from 'The Cytochrome P450 homepage' reported by D. R. Nelson (http://drnelson.uthsc.edu/CytochromeP450.html) [21]. These 254 Arabidopsis P450 sequences were treated as a query to perform BlastP search with the E-value $\leq 1 \mathrm{e}^{-}$ 40 against tomato (Solanum lycopersicum) genome (ITAG2.3) available at Phytozome database V10 (www.phytozome.net) [18]. Furthermore, a manual analysis of putative Solanum lycopersicum P450 (SlP450) sequences was conducted for the complete ORF and truncation. The analysis consist of non-redundant and full-length SlP450 genes. Universal names for SlP450 genes were assigned according to the standard system of P450 nomenclature committee [20, 21].

\section{Multiple sequence alignment, phylogenetic tree construction and conserved motif analysis}

The 48 P450 protein sequences from other plants such as Arabidopsis thaliana (40), Populas trichocarpa (1) and Solanum tuberosum (7) along with 233 SlP450s from Solanum lycopersicum were considered to construct the phylogenetic tree. The accession numbers are provided in Additional file 1. Multiple sequence alignment of these P450 genes was carried out with Muscle algorithm [22] using default parameters present in MEGAX software [23]. The phylogenetic tree was constructed using Neighbour-joining (NJ) [24] and maximum likelihood (ML) algorithm. The Dayhoff substitution matrix (PAM250) along with the bootstrapping (1000 replicates) was employed for NJ analysis. The unrooted maximum likelihood phylogenetic tree and evolutionary analyses were carried out using IQ-TREE web server (http://iqtree.cibiv.univie.ac.at/) [25]. The best-fit model was selected from 168 amino acid substitution models using modelfinder tool [26]. The modelfinder reported $\mathrm{LG}+\mathrm{F}+\mathrm{I}+\mathrm{G} 4$ as best-fit model according to bayesian information criterion (BIC score 420,547.05). The ML tree was built with 1000 ultrafast bootstrap [27] replications and the final tree with highest $\log$ likelihood $(-208,278.21)$ was considered for 
phylogeny inferences. For conserved domain identification, multiple sequence alignment of $S l P 450$ protein sequence were carried out using Clustal X program using default parameters [28]. The alignment file was submitted to Web Logo generator software for generating the logo of conserved domains available at (http://weblogo.berkeley.edu/) [29].

\section{Intron map and their organization}

Intron map of tomato P450 genes was drawn by using previously described methods suggested by Barvkar et.al. and Paquette et.al. [30, 31]. The intron-exon boundaries, introns phases and their position in protein sequences were considered for the same. Introns present in genomic sequences, were mapped on protein sequences and serially numbered. Introns can have three intron phases: intron phase 0,1 and 2 . Introns with the identical positions in one codon along with similar intron phase are termed as 'conserved intron'. The intron map was constructed by considering 145 (62.23\%), SlP450 genes sequences with one and two introns.

\section{Promoter analysis of S/P450 genes and identification of tissue specific promoters}

The promoter analysis of tomato P450 genes helps to identify over-represented motifs regulating gene expression. We used previously characterized motifs from PLACE [32] and plant CARE databases [33] to obtain regulatory motifs which are over-represented in a group of genes. The consensus motifs from these databases were used since it has high coverage of previously characterized plant motifs (total 946 plant motifs). The complete Solanum lycopersicum genome was downloaded from Phytozome database. Moreover, the bed file with genomic coordinates was used to extract $2 \mathrm{~kb}$ upstream sequence of all the protein coding genes using bedtools suite with getfasta option [34]. The promoter motifs for all protein-coding genes were identified using perl script generously shared by Dr. Angelica Lindlöf [35]. The presence of core promoter sequence can occur randomly because of the short length. Hence, we excluded random occurrence probability of any promoter motif in SlP450 upstream sequence. To calculate non-random occurrence probability, the presence or absence of individual promoter motif in two groups was compared statistically. The first group included SlP450 genes highly expressed in specific tissue types (Leaf, buds, peel, petals, roots, seeds) and the second group contains all the protein coding genes. The statistical one-sample test for binomial proportions was applied at significant $p$-value $(\leq 0.05)$. We used fragments per kilobase of transcript per million mapped reads (FPKM) values from RNA sequencing of various tissue types to understand the relationship between promoter occurrence and actual gene expression of individual SlP450 gene. Furthermore, a comparison was carried between previously mentioned two groups. The motifs which are statistically significantly overrepresented were assigned as tissue specific promoter motif that are driving expression of selected SlP450 genes.

\section{Digital expression analysis of S/P450 genes}

The digital expression analysis was performed to gain an insight of the role of the identified SlP450 in the various tissues. We used publicly available RNA-sequencing data from Dr. Asaph Aharoni lab (https://www.weizmann.ac.il/plants/aharoni/sites/plants.aharoni/files/uploads/ tomato_rnaseq_data_19_tissues.xlsx) in order to decipher expression of SlP450 in 19 different tissues namely leaf, root, floral buds, petals and peel, flesh, seeds of immature green, mature green, breaker, orange and red fruits respectively. Available RNA-sequencing data were normalized with FPKM method. Digital expression profile of SlP450 genes in the form of heat map was constructed using ClustVis software (http://biit.cs.ut.ee/ clustvis/) with default parameters [36].

\section{Plant material}

The Solanum lycoperscium L. cv MicroTom (TGRC accession number: LA3911) seeds were generously provided by Prof. Asaph Aharoni (Department of Plant Sciences, Weizmann Institute of Science, Israel) which were obtained from Tomato Genetics Resource Center (http://tgrc.ucdavis.edu). The Tomato plants were grown in the poly house and maintained at controlled conditions of temperature $\left(25^{\circ} \mathrm{C}\right)$ and humidity $(54 \%)$. On maturation of plants, root $(\mathrm{R})$, stem $(\mathrm{S})$, leaves $(\mathrm{L})$, flower (F), green fruit (GF), mature green fruit (MGF) tissues were harvested. The tissues were frozen in liquid nitrogen and stored at $-80^{\circ} \mathrm{C}$ until further use.

\section{Real-time quantitative PCR (RT-qPCR) analysis}

To confirm the digital expression analysis of SlP450s, we have selected six genes i.e. SlCYP51G1, SlCYP90A5, SlCYP77A20, SlCYP71AX11, SlCYP74C3 and SICYP733A depending on their higher expression in various tissues. Total RNA from root, stem, leaves, flower, green fruit, and mature green fruit tissues were extracted using trizol reagent (Invitrogen, USA) [37] as per the manufactures protocol. Total RNA was quantified with NanoDrop (ND-1000 spectrophotometer, Wilmington, USA) and then treated with RNase-free DNaseI (Promega, USA) to remove DNA contamination. Total $2 \mu \mathrm{g}$ of RNA was reverse transcribed into cDNA by using AMV reverse transcriptase (Applied biosystems, USA) [38]. The cDNA synthesized from different tissues were used for RT-qPCR analysis. Primers for RT-qPCR were designed using Primer 3 software 
available at (http://bioinfo.ut.ee/primer3-0.4.0/). The primer sequences are available in the Additional file 2 . RT-qPCR analysis was performed in the Realflex2 Master cycler (Eppendorf, Germany). We used $5 \mu \mathrm{l}$ of $2 \mathrm{x}$ SYBR green master mix (Roche, USA), sterile milliQ water, 10pM forward and reverse primer and 1.5 $\mu \mathrm{l}(1: 3$ diluted) cDNA for RT-qPCR analysis. Thermal profile used for RT-qPCR analysis were as follows: initial denaturation at $95^{\circ} \mathrm{C}: 5 \mathrm{~min}$ followed by $95^{\circ} \mathrm{C}: 15 \mathrm{~s}, 60^{\circ}$ C: $30 \mathrm{~s}, 72^{\circ} \mathrm{C}: 30 \mathrm{~s}$ for 40 cycles. After amplification, melting curve analysis was conducted at $60-95^{\circ} \mathrm{C}$ ramps with $0.5{ }^{\circ} \mathrm{C}$ increment per cycle to check the primer spe-

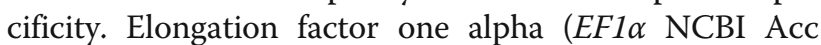
No. NM_001247106) gene was used as housekeeping/internal control after verifying the uniform expression in all the studied tissues of tomato. Relative expression profile of selected six candidate genes SlCYP51G1, SlCYP90A5, SlCYP77A20, SlCYP71AX11, SlCYP74C3, SlCYP733A were determined by using 2(-Delta Delta $\mathrm{C}(\mathrm{T})$ ) Method as described by Livak et al. [39]. Each gene had a PCR efficiency and $R^{2}$ value between $0.9-$ 1.00 along with single melting curves. The experiment was repeated with three biological and two technical replicates for each gene.

\section{Results}

\section{Annotation and classification of tomato P450 multigene family}

A total of 300 tomato P450 genes were identified from tomato genome which includes full length, pseudo genes and truncated genes. Moreover, 233 putative non redundant full length P450 gene sequences were identified using BlastP search. All four conserved key motifs i.e. heme binding domain, I-helix, K-helix and PERF/W motif were part of it. These sequences possess complete ORF and amino acid length that varies from 450 to 600 residues with an average of 505 amino acids. The average percent identity of 233 SlP450 proteins was 25.87 and ranges from 95.7 to 13.7. The isoforms of 94B (SlCYP94B18 and SlCYP94B20) showed maximum percent identity, whereas pair SlCYP74C4 and SlCYP701A30 exhibited minimum percent identity (Additional file 1). Four conserved motifs are shown in the Fig. 1 . These are similar as previously described by Bak et al. [5].

\section{Phylogenetic analysis of the tomato P450 multigene family}

The phylogenetic tree of SlP450 proteins divided into two major clades: A-type and non-A type. These two clades are further clustered into nine clan i.e. clan71, clan51, clan710, clan85, clan711, clan86, clan97, clan72, and clan74 [Fig. 2]. The tree topology of NJ and ML tree [Additional file 3] is similar therefore, it indicate the robustness of phylogenetic tree and clustering of SlP450 genes into families and clans. Phylogenetic analysis revealed that clan51, clan710, clan711 and clan74 are single family clans; remaining five clans contain multiple families of SlP450 genes [40, 41]. Overall SlP450 genes are classified into 42 families. The 137 (59\%) SlP450 genes are designated as A-type and can further be divided into 21 families while 96 (41\%) SlP450 genes are assigned as non-A type and can be classified into 21 families. In tomato genome clan71 comprises more than $50 \%$ genes. The CYP71 family is largest A-type family which contains 43 (31.61\%) genes divided into 10 subfamilies i.e. CYP71D, CYP71AH, CYP71AT, CYP71AU, CYP71AX, CYP71BG, CYP71BE, CYP71BL, CYP71BN and CYP71BP. The clan 72 has eight subfamilies whereas CYP72 is the largest non-A family which contains 20 (20.83\%) genes. It is further divided into two subfamilies namely CYP72A and CYP72D [5]. During the course of evolution CYP728, CYP733, CYP80, CYP92, CYP736 and CYP749 families were evolved in tomato genome and lost from Arabidopsis genome. $S l C Y P 51, S l C Y P 710$ and SlCYP85 clans cluster together in the phylogenetic tree indicating paralogous origin. SlCYP74 clan has four subfamilies and act as outgroup in the phylogenetic tree since it is an atypical plant P450 clan which lacks the monooxygenase activity. The clan 97 and 86 appears to share common ancestral genes and therefore they are clustered together in the phylogenetic tree.

\section{Intron gain and loss events to investigate evolution of P450 multigene family}

Understanding gain and loss of the intron reflects the evolution of gene family. In the present study, we analysed the intron number and phases. The identified SlP450 genes have minimum zero and maximum 14 introns. Out of 233 SlP450 genes, 23 (9.87\%) genes have no intron, 108 (46.35\%) genes have one intron, 37 (15.87\%) genes have two introns, four genes $(1.71 \%)$ have three introns, 30 genes (12.87) have four introns and 31 genes $(31.30 \%)$ contain five/ more than five introns. The intron map of P450 gene sequences was constructed by considering 145 genes that had one and two introns (comprising $62.23 \%$ of the total genes). The data used to construct the intron map and distribution graph are provided in Additional file 4. A total of 22 independent intron insertion events were occurred in SlP450 genes [Fig. 3]. If intron position in a particular sequence was within 40-45 amino acids of its mean recorded position across the sequences, it was considered as conserved [30]. Introns number I13 and I14 are conserved in intron map. Intron map analysis revealed that most of the gene families contain conserved intron I13 (56.55\%) and I14 (17.93\%). These two introns are recent introns amongst identified 22 introns. Both conserved introns 

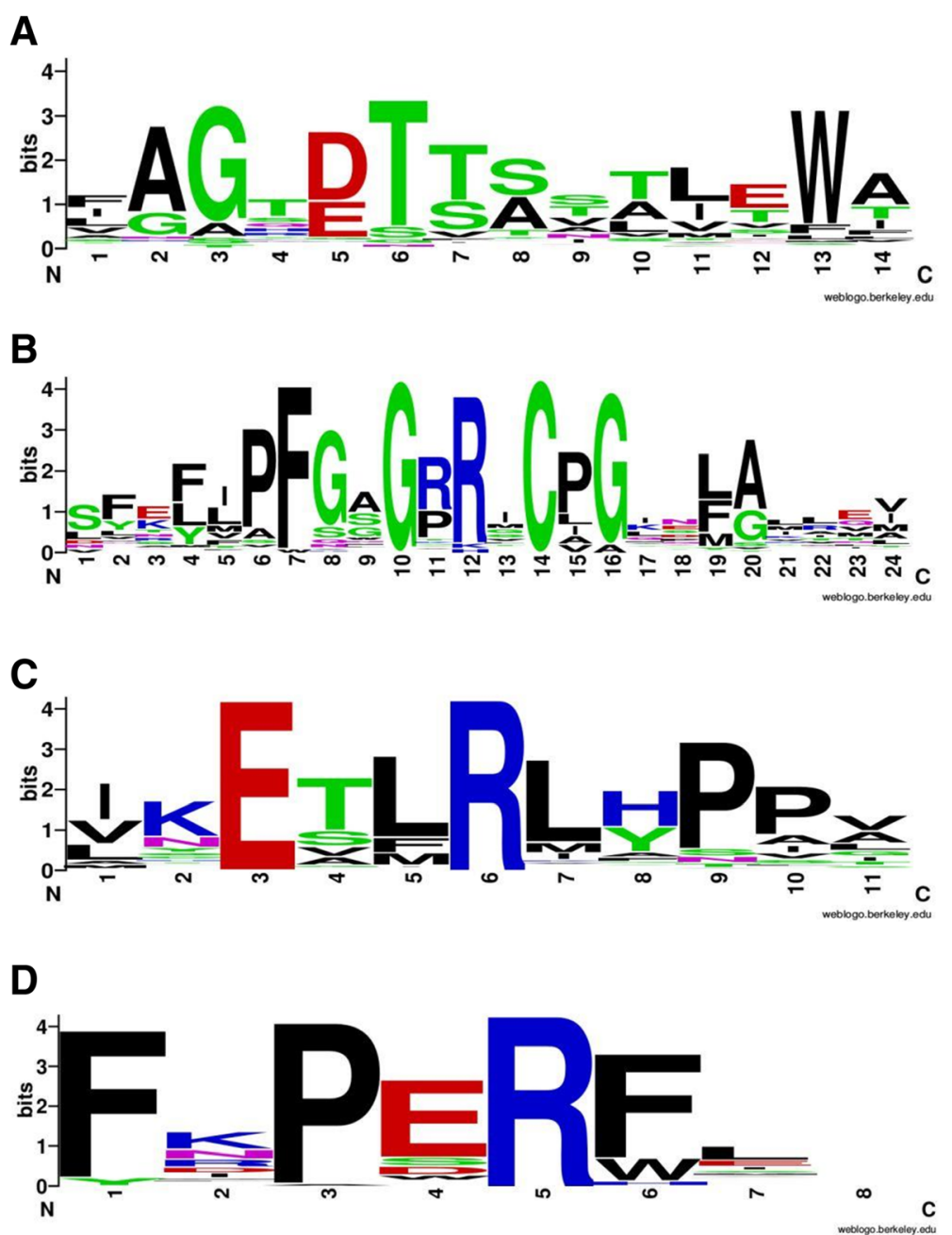

Fig. 1 Conserved motifs/sequence logos of the predicted tomato P450 proteins: Web Logos of conserved motifs in P450 from tomato, A. thaliana, P. trichocarpa and S. tuberosum P450 sequences. Letter size in the logos is proportional to the degree of conservation. a AGXDT (I- helix), (b) Heme binding motif, (c) KETLR (K-helix), (d) PERF/W motif respectively

are present in gene families belonging to clan71. Families with conserved intron I13; lack conserved intron I14 and vice versa. For example, CYP84 family gene has conserved intron I13 whereas it lost the conserved intron I14 and contains additional intron at I2 insertion site. It was observed that I13 intron has evolved during the course of evolution and I14 intron was lost from SlP450 genes (Fig. 3). In the intron map, 122 (84.13\%) genes have conserved intron I13 and the remaining genes have conserved intron I14 at intron insertion site. Out of 145 genes, 106 genes have intron phase one and 39 genes have intron phase zero and two. It was observed that gene families from same phylogenetic group have similar intron numbers and organization. The SlP450 genes belonging to non-A type families lack conserved introns but have introns at different intron insertion sites. For example, SlCYP51 from clan51 lost both the conserved introns, gained I5 intron and created separate family. The
SlCYP718A6 and family SlCYP716 genes that belongs to clan85 also lost both the conserved introns, gained I18 intron and diverged. Both the conserved introns were in the same intron phase and only appear in A-type P450 clan. This suggests the recent diversification of A-type P450 genes from common ancestral gene and neofunctionalization during the course of evolution [30].

\section{In silico analysis of tomato $\mathrm{P} 450$ gene promoters}

Promoter motifs play crucial role in execution of the biological functions of the genes. The comparisons were carried out between group of SlP450 which were highly expressed in different tissue types with all the protein coding genes in tomato. The list of over-represented motifs obtained from promoter analysis of 233 tomato P450 genes are enlisted in Additional file 5. Among 233 SlCYPs, 73 (31.33\%) genes which had digital expression evidence were considered for further promoter analysis. 


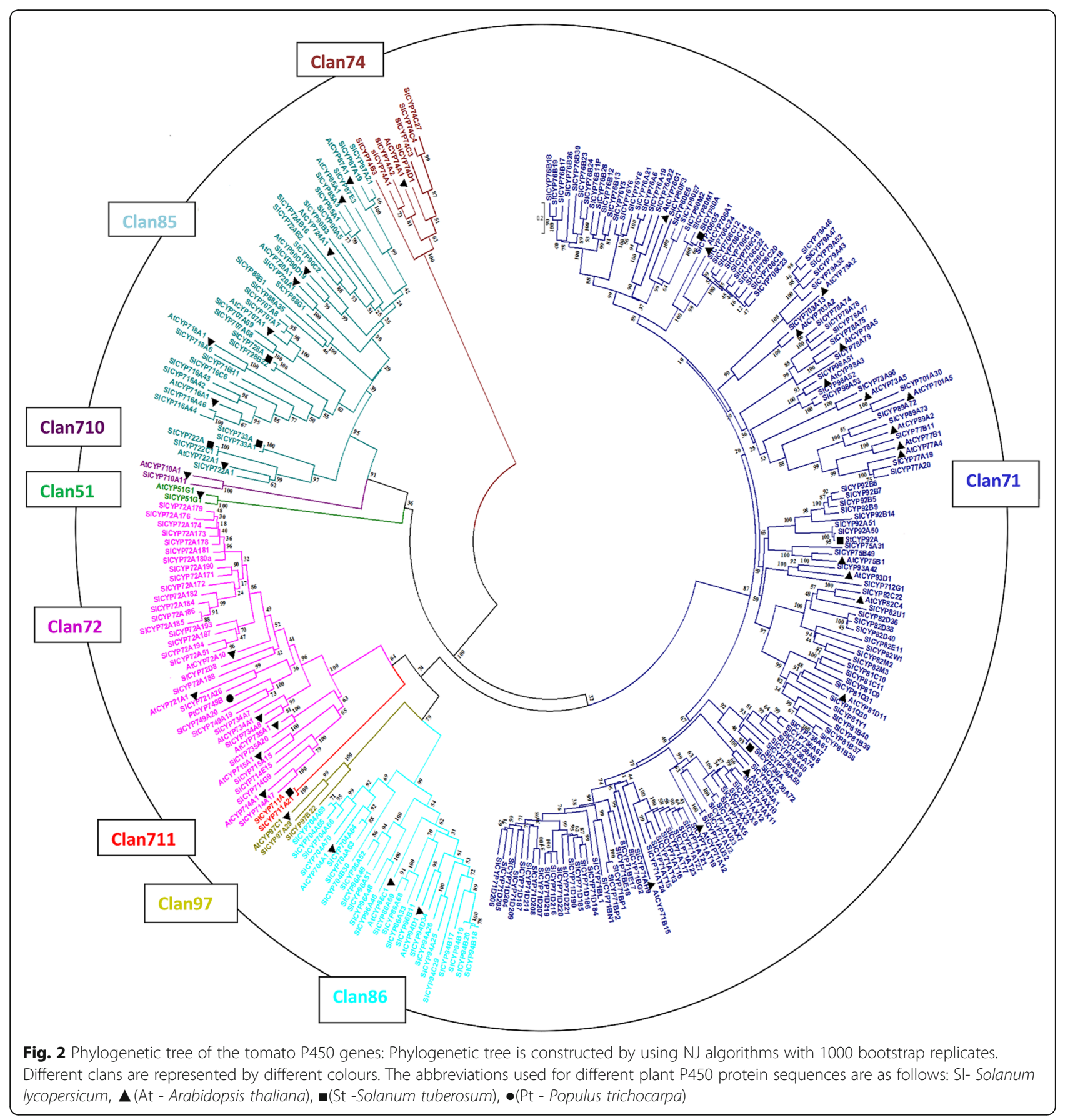

Specific over-represented promoter motifs from selected tomato tissues specific P450 gene are summarised in Table 1. with their probable biological function.

\section{Digital expression profiling of tomato P450 genes}

The FPKM normalized expression values were used to construct digital expression profile heat map [Fig. 4]. Among 233 SlP450 genes, 73 (31.33\%) genes were differentially expressed in different tissues. The developing seeds from different fruits ripening stages show large proportion $(72.60 \%)$ of highly expressed P450 genes whereas least number of genes are expressed in buds (5.47\%). Phylogenetic family specific expression of P450 genes varies from 2.38 to 930.98 FPKM (Additional file 6). Moreover, the digital expression was validated by RT-qPCR analysis of six candidate SlP450genes that represented both single gene family and multigene family clades of tomato P450. These selected P450 were analysed for their relative transcript abundance and are graphically represented in Fig. 5. The SlCYP51G1 


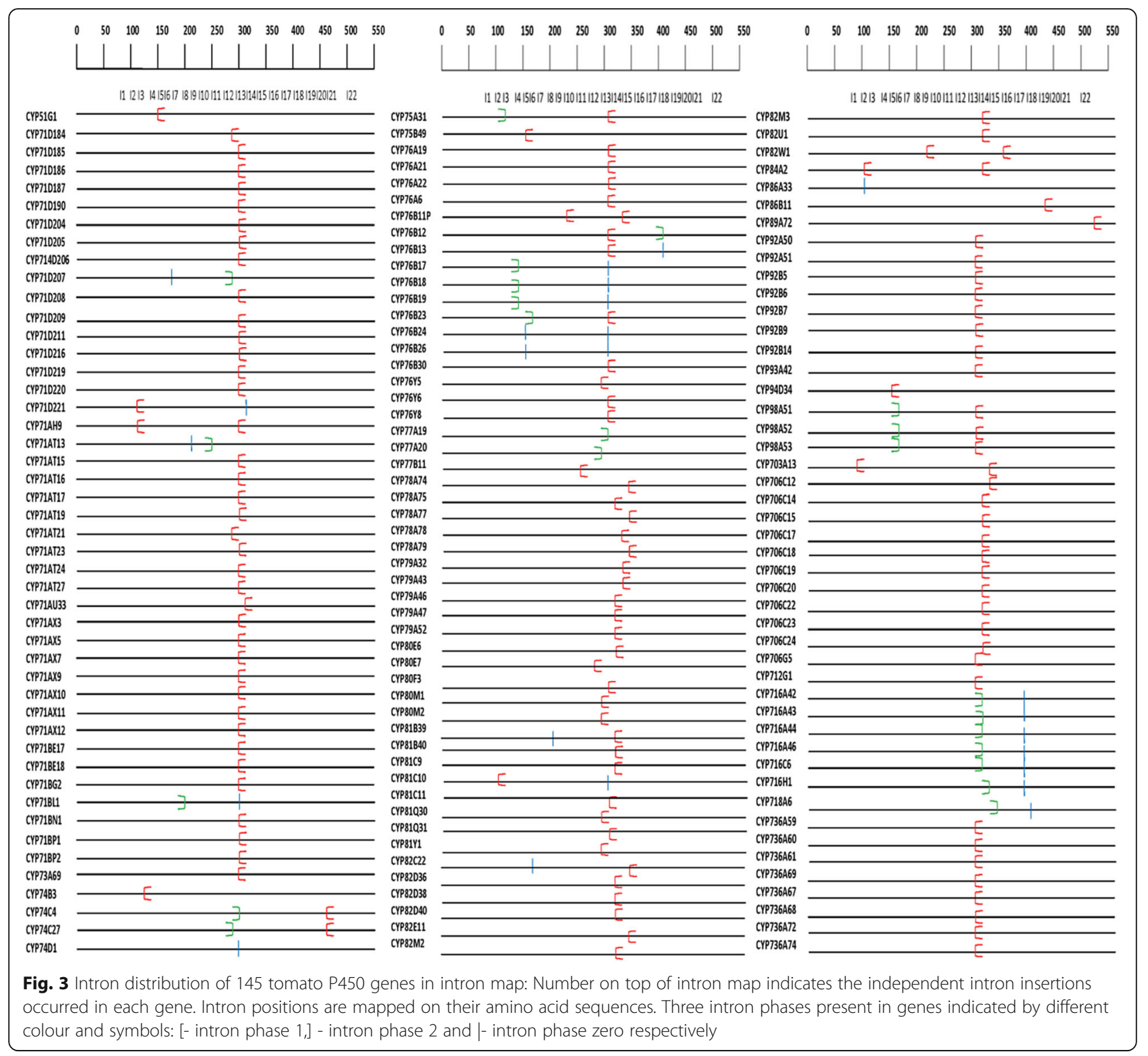

exhibited 0.29 fold higher relative transcript abundance in flower. The SlCYP77A20 and SlCYP90A5 had 0.39 and 0.15 fold relative transcript upregulation in green fruit and flower, respectively which were corroborated with RNA sequencing data. The SlCYP71AX11 showed 0.031 fold expression in mature green fruit. SlCYP74C3 and SlCYP733A1 genes had 0.005 and 0.094 fold relative transcript abundance in leaf and flower. The RT-qPCR analysis results were correlated with RNA sequencing data.

\section{Discussion}

Cytochrome P450 genes are involved in catalysis of variety of reactions which include growth, development and secondary metabolite biosynthetic pathways. In present study we identified 233 P450 genes from tomato which are comparable with genes identified in Arabidopsis thaliana (245) [5] but more than mulberry (176) [15]. All identified tomato P450 genes contain four P450 signature conserved domains. The orthologs comparison of tomato P450 gene families with plant species such as Arabidopsis, Medicago, poplar, flax, moss, rice and soybean revealed the evolution of P450 gene family (Additional file 7). These results demonstrated that CYP702 and CYP708 families are present in Arabidopsis and absent from other analysed plants. This may be attributed to biosynthesis of triterpenoid derivatives that are Brassicaceae specific [57]. The CYP749A20 gene was up-regulated in red and orange fruit with unknown function in tomato. However, its orthologue from 
Table 1 Tissue specific SIP450 having over-represented promoter motifs along with their probable biological role

\begin{tabular}{|c|c|c|c|c|c|}
\hline $\begin{array}{l}\text { Sr. } \\
\text { No }\end{array}$ & $\begin{array}{l}\text { Over } \\
\text { represented } \\
\text { Motif name }\end{array}$ & $\begin{array}{l}\text { Tomato } \\
\text { Tissue Type }\end{array}$ & Biological function & Solyc Id & $\begin{array}{l}\text { Universal } \\
\text { name }\end{array}$ \\
\hline 1 & $\begin{array}{l}\text { AC motif and } \\
\text { MYB1LEPR motif }\end{array}$ & $\begin{array}{l}\text { Leaf specific } \\
\text { P450 genes }\end{array}$ & $\begin{array}{l}\text { These motifs are present in bean phenylalanine ammonia-lyase (PAL) gene and } \\
\text { together play crucial role to co-ordinate regulation of phenylpropanoide metab- } \\
\text { olism }[41,42,43]\end{array}$ & $\begin{array}{l}\text { Solyc04g071800 } \\
\text { Solyc11g006590 } \\
\text { Solyc04g011690 }\end{array}$ & $\begin{array}{l}\text { SICYP92B7 } \\
\text { SICYP71AT7 } \\
\text { SICYP736A }\end{array}$ \\
\hline 3 & AGL motif & $\begin{array}{l}\text { Root specific } \\
\text { P450 genes }\end{array}$ & $\begin{array}{l}\text { Arabidopsis AGL19 and AGL18 promoter motif showed specific expression in } \\
\text { root meristem and central cylinder cell in mature root and also in petals and } \\
\text { siliques }[44,45,46,47] \text {. }\end{array}$ & $\begin{array}{l}\text { Solyc12g042480 } \\
\text { Solyc02g084570 } \\
\text { Solyc03g120060 } \\
\text { Solyc10g017510 } \\
\text { Solyc05g047680 }\end{array}$ & $\begin{array}{l}\text { SICYP736A4 } \\
\text { SICYP84A2 } \\
\text { SICYP734A8 } \\
\text { SICYP71BE8 } \\
\text { SICYP78A77 }\end{array}$ \\
\hline 4 & AT-box motif & $\begin{array}{l}\text { Buds specific } \\
\text { P450 genes }\end{array}$ & $\begin{array}{l}\text { AT rich binding sequence characterized from promoter of tomato rbcs-3A gene. } \\
\text { This motif meditate regulation of light harvesting gene complex }[48,49,50] \text {. }\end{array}$ & $\begin{array}{l}\text { Solyc04g078900 } \\
\text { Solyc12g006460 } \\
\text { Solyc07g043460 }\end{array}$ & $\begin{array}{l}\text { SICYP707A8 } \\
\text { SICYP88B1 } \\
\text { SICYP72A18 }\end{array}$ \\
\hline 5 & $\begin{array}{l}\text { Auxin } \\
\text { responsive } \\
\text { element }\end{array}$ & $\begin{array}{l}\text { Root specific } \\
\text { P450 genes }\end{array}$ & $\begin{array}{l}\text { Soybean GH3 gene has three auxin responsive element which are important in } \\
\text { auxin mediated gene expression [51]. }\end{array}$ & $\begin{array}{l}\text { Solyc } 12 g 042480 \\
\text { Solyc02g084570 } \\
\text { Solyc03g120060 } \\
\text { Solyc10g017510 } \\
\text { Solyc05g047680 }\end{array}$ & $\begin{array}{l}\text { SICYP736A4 } \\
\text { SICYP84A2 } \\
\text { SICYP734A8 } \\
\text { SICYP71BE8 } \\
\text { SICYP78A77 }\end{array}$ \\
\hline 6 & $\begin{array}{l}\text { HSE heat shock } \\
\text { element }\end{array}$ & $\begin{array}{l}\text { Root specific } \\
\text { P450 genes }\end{array}$ & $\begin{array}{l}\text { HSE are present in the heat shock proteins of Apx } 1 \text { gene and involved in } \\
\text { oxidative stress defense. Arabidopsis APX1 gene showed induced expression } \\
\text { under oxidative stress }[52,53] \text {. }\end{array}$ & $\begin{array}{l}\text { Solyc12g042480 } \\
\text { Solyc02g084570 } \\
\text { Solyc03g120060 } \\
\text { Solyc10g017510 } \\
\text { Solyc05g047680 }\end{array}$ & $\begin{array}{l}\text { SICYP736A4 } \\
\text { SICYP84A2 } \\
\text { SICYP734A8 } \\
\text { SICYP71BE8 } \\
\text { SICYP78A77 }\end{array}$ \\
\hline 7 & $\begin{array}{l}\text { TCP } \\
\text { transcription } \\
\text { factor }\end{array}$ & $\begin{array}{l}\text { Petal specific } \\
\text { P450 genes }\end{array}$ & $\begin{array}{l}\text { TCP transcription factor involved in growth, development and defense } \\
\text { mechanism also induces biosynthesis of Brassinosteroid (BR), Jasmonic acid (JA) } \\
\text { and flavonoids might be involved in regulation of floral tissues developing } \\
\text { genes in tomato plant. In Arabidopsis TCP14 and TCP15 motifs are involved in } \\
\text { regulation of floral tissues and leaf blade development }[54,55,56] \text {. }\end{array}$ & $\begin{array}{l}\text { Solyc04g050620 } \\
\text { Solyc07g062500 } \\
\text { Solyc06g051750 } \\
\text { Solyc08g079280 } \\
\text { Solyc04g051190 } \\
\text { Solyc08g080380 } \\
\text { Solyc02g080330 } \\
\text { Solyc10g080870 } \\
\text { Solyc09g059240 } \\
\text { Solyc11g065770 }\end{array}$ & $\begin{array}{l}\text { SICYP736A1 } \\
\text { SICYP72A14 } \\
\text { SICYP90A5 } \\
\text { SICYP706C2 } \\
\text { SICYP97A29 } \\
\text { SICYP80E6 } \\
\text { SICYP77B11 } \\
\text { SICYP96A48 } \\
\text { SICYP82U1 } \\
\text { SICYP94A25 }\end{array}$ \\
\hline
\end{tabular}

Arabidopsis is absent. During the course of evolution, CYP749 family is evolved only in Asteroids, Rosides and Ranunculales members [5]. Tomato CYP78 family members have only CYP78A subfamily, interestingly genes from this family are involved in flower development and meristem specific function in Arabidopsis [5]. The SlCYP78A sub-family genes, SlCYP78A75 and SlCYP78A77 were respectively up-regulated in flower buds and root. In addition, the SlCYP78A77 also contains root specific promoter motifs i.e. auxin responsive element and HSE (heat shock element). These motifs are consequently involved in auxin mediated gene expression and combating oxidative stress in other plants [51, 52, 53]. The SlCYP81 family has 10 genes distributed in four sub families which belong to clan71. The SlCYP81B and SlCYP81C subfamily genes were up-regulated during different stages of the tomato fruit development. It is demonstrated in Arabidopsis that CYP81D, CYP81F, CYP81H and CYP81G subfamily genes play important role in disease resistance $[57,58]$. The SlCYP81B and SlCYP81C might be involved in tomato fruit development as well as protection from different diseases since they are highly expressed in these tissue types [41]. The
CYP80 family is present in tomato, poplar and grape. It supposedly involved in phenolic coupling during alkaloid biosynthesis [59]. The SlCYP8OE6 gene found to be up-regulated in petals and it contain overrepresented TCP transcription factor which was a petal specific motif. In Arabidopsis, TCP transcription factor is involved in floral organs development and biosynthesis of different phytohormones $[54,55,56]$. Hence, SlCYP80E6 is a potential candidate to study the floral development. The expression data suggests that SlCYP84A2 gene was up-regulated in root and has root specific overrepresented AGL promoter motif. In Arabidopsis, CYP84A1 gene is involved in the lignin biosynthesis. The functional analysis of this gene affects the lignification and vascular development [5]. Expression and promoter data from tomato suggests that SlCYP84A2 gene might be involved in vascular development of the root.

Phylogenetic tree topology of tomato and Arabidopsis P450 revealed similar clustering that indicates conserved nature of $\mathrm{P} 450$ multigene family across the various plant species [20]. The single family clans contain low copy genes with essential function in all the plants. They restrict themselves from gene duplication due to strong 


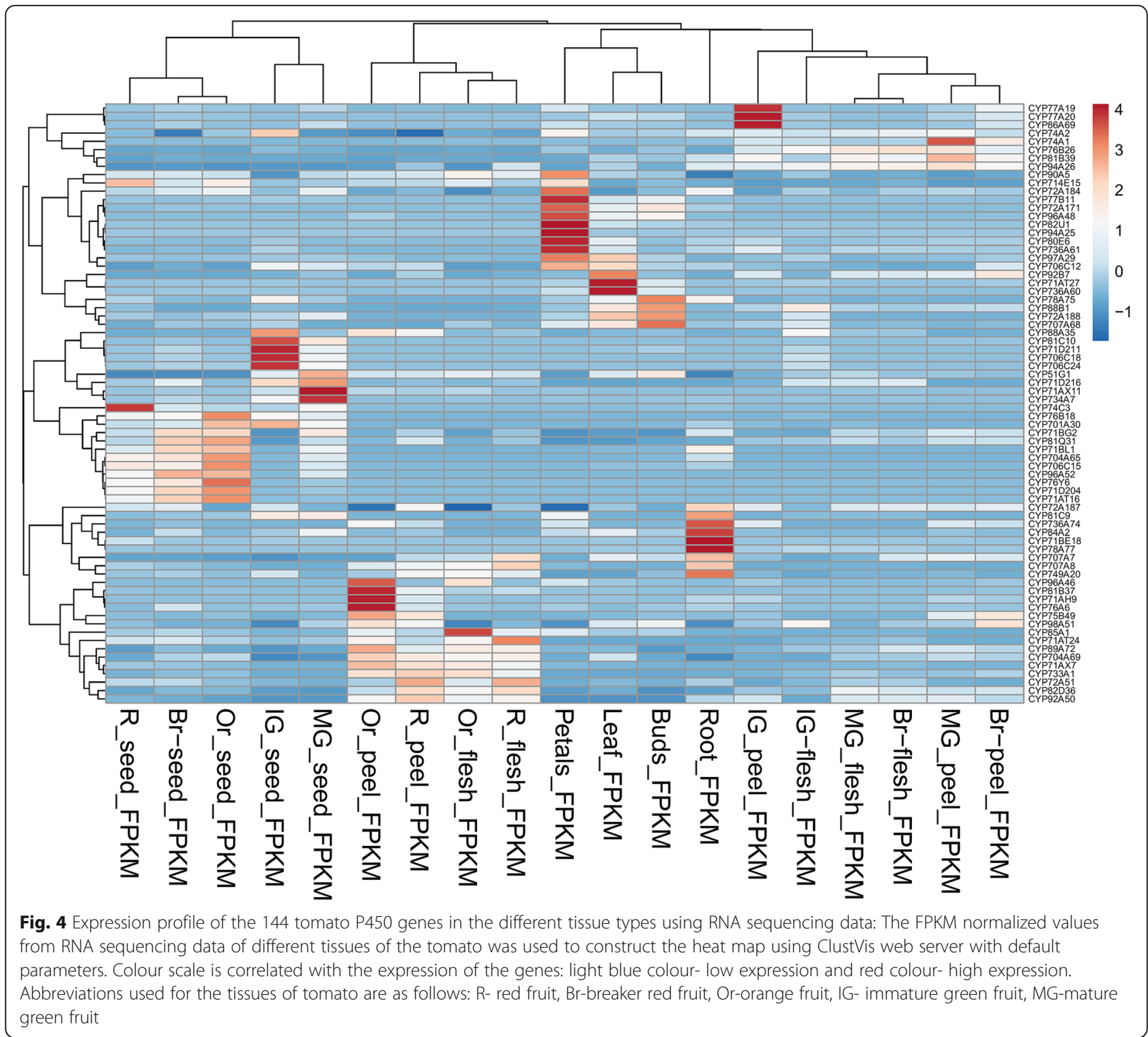

purifying/negative selection process [60]. The CYP51 is ancient and conserved clan with single copy in all the phyla studied so far. The SlCYP51G1 showed $82 \%$ identity with AtCYP51G1 and it is involved in sterol metabolism [60]. RT-qPCR expression data has showed that SlCYP51G1 is constitutively expressed in all selected tissues of tomato and has sterol demethylase activity required for the maintenance of membrane integrity [61]. The CYP71 family genes evolve through the gene duplication and seems to be recent in the evolutionary history $[60,61]$. Tomato CYP71 family genes have average 30\% sequence identity with Arabidopsis CYP71 family. The expression data of SlCYP71AX and SlCYP77A20 from to clan71 showed that these two genes were up-regulated in green and mature green fruit of tomato which is in accordance with the transcriptome data. These two genes would be good candidates for the study of secondary metabolite synthesis in tomato fruit $[62,63]$.

The CYP74 family is an atypical plant P450 family and thought to be involved in catalysis of already oxygenated polyunsaturated $\mathrm{C} 18$ fatty acid hydroperoxide into other oxylipins [5]. The RT-qPCR data exhibited upregulation of SlCYP74C3 gene in mature green tomato fruits and hence it could be a potential candidate gene to study oxylipin biosynthesis in tomato fruit. The SlCYP90A5 gene was up-regulated in tomato flower and showed less expression in leaf which correlate with transcriptome data. The AtCYP90A1 is involved in brassinosteroid metabolism and shows less expression in expanding leaf $[58,5]$ whereas tomato orthologue SlCYP90A5 has similar expression profile. CBF/DREB1 transcription factor plays role in cold response [64] and is over-represented 
CYP51G1

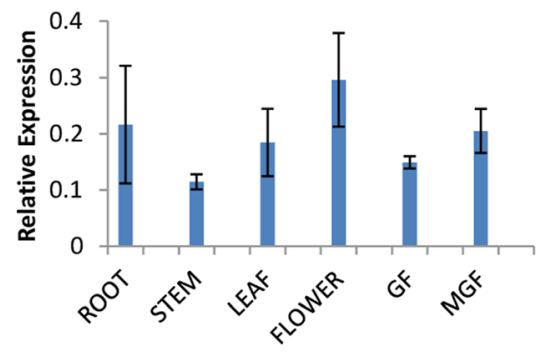

CYP74C3

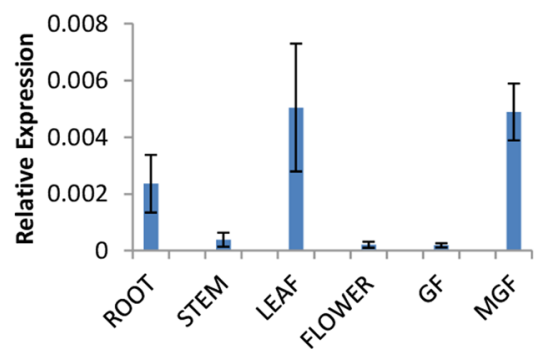

CYP90A5

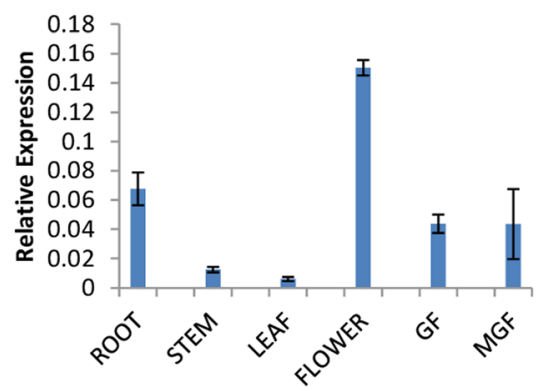

CYP71AX11

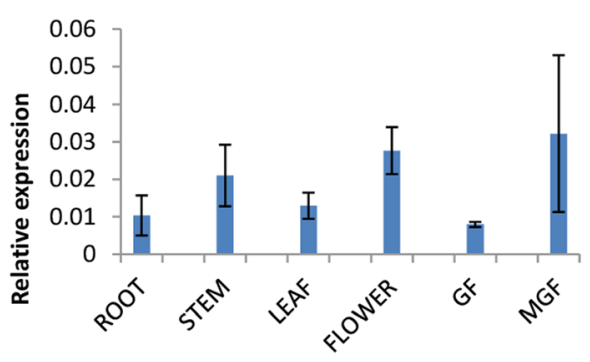

CYP77A20

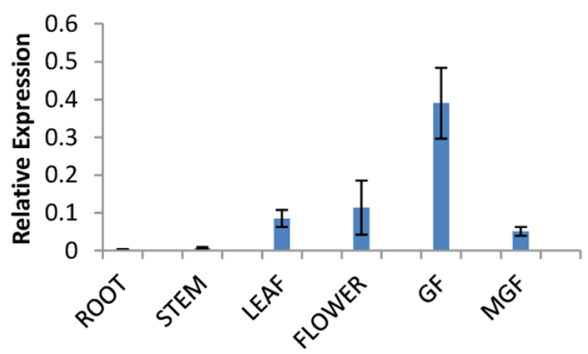

CYP733A1

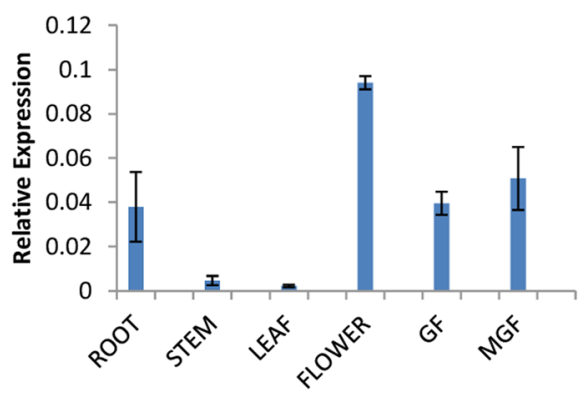

Fig. 5 Expression of the selected candidate P450 genes in different tissue types: X-axis represents the different tissue types and $y$-axis denotes the relative expression values for the different genes. Bar indicate the mean value and Error bar indicates the standard deviation of the three biological replicates. Abbreviations used in graph for various tissues are as follows: GF- immature green fruit, MGF- mature green fruit

in SlCYP72A184, SlCYP85A1 and SlCYP96A48 genes. These genes can be candidate for cold stress tolerance in tomato. Intron map along with their phases and gain/ loss events plays a crucial role in understanding the evolution of gene families within phylogenetic group. Conserved introns are ancient elements and present with similar intron phase [65]. Intron phase changes due to intron sliding events or changing intron-exon boundaries with one or two nucleotides [66]. Introns tend to maintain their phases during evolution, given that changes in intron phases occur rarely. In the mulberry P450, maximum genes contain one and two introns that were comparable with tomato P450 introns [15]. Both conserved introns were evolved in clan71 gene families due to gene duplication events. In Arabidopsis, two conserved introns were absent from non-A type $\mathrm{P} 450$ gene families whereas they appeared in A-type P450 gene families [31]. It is observed that conserved intron I13 evolved gradually and conserved intron I14 lost from SlP450 genes during the course of evolution. Intron gain was observed in the A-type of SlP450 genes which was absent in the ancestral (Non-A) gene families. Hence, this data support the intron late view of intron evolution [30, 31].

The expression evidence to the genes profoundly depends on developmental stages, age of the plant, environmental conditions, extent of expression, tissue specificity and biotic or abiotic stress. In the present 
study, only $31.33 \%$ P450 genes showed evidence of expression which could be compared with rice (49.81\%) [67] and soybean (31.92\%) [14]. In mulberry, Ma et.al. (2014) have identified 173 P450 genes which were further divided into five clusters for expression profile and found that the maximum 23.6\% P450 genes were expressed [15]. Present study is conducted on the available RNA sequencing data of different tissues of tomato. The data was not obtained by challenging plant with any pathogen or exposing plants to the different stress conditions. Following possibilities can be asserted in the given case: i) remaining genes have developmental specificity or ii) it is expressed in different biotic or abiotic stress conditions or iii) it is present in the in-detectable level or iv) is inactive. The digital expression analysis provides global landscape could be instrumental to study various tissue specific P450. The promoter analysis suggested SlP450 promoter motifs are driving tissue specific expression. Thus present study may enable researchers to select appropriate candidate gene from huge repertoire of SlP450 for detailed functional characterization.

\section{Conclusion}

The Tomato genome has a greater number of P450 clans as compared to Arabidopsis with variable number of P450 genes in each clan. Phylogenetic tree analysis provided the information about the functional evolution of P450 gene family in tomato. In intron map, gain and loss of conserved introns reveals P450 gene family evolution in tomato plant. Digital and experimental expression profile suggests tissues specific highly expressed P450 genes that could be potential candidates for further study. The promoter motifs driving the higher expression of $\mathrm{P} 450$ in analysed tissues types can be further evaluated using functional genomics for traits of economic importance. Thus, this study provides solid foundation for functional characterization of candidate genes with their biological significance.

\section{Additional files}

Additional file 1: Table of SIP450 summary file 233 SIP450 sequences (genomic, transcript, CDS and protein) are provided with the universal names, Sol genomics id and phylogenetic groups. The NCBI accession numbers of Arabidopsis, Poplar and Potato P450 protein sequences used in phylogeny are listed. The sheet 2 represents percent identity matrix of all the SIP450 proteins. (XLSX $197 \mathrm{~kb}$ )

Additional file 2: Table of primer sequences used in experimentation. Primer sequences of genes used RT-qPCR analysis. (XLSX 8 kb)

Additional file 3: SIP450 phylogenetic tree inferred using Maximum likelihood method. Phylogenetic tree is constructed by applying maximum likelihood method with 1000 ultrafast bootstrap replicates using $L G+F+I+G 4$ as best-fit substitution model. (TIF 13827 kb)

Additional file 4: Intron analysis summary. Data for Intron map constructions and distribution. (XLSX $34 \mathrm{~kb}$ )
Additional file 5: Promoter analysis Table. Promoter analysis of all the protein coding genes in tomato with promoter motifs count. (XLSX $81994 \mathrm{~kb})$

Additional file 6: Tissue specific expression of P450 with FPKM count. Highly expressed tomato P450 genes from heat map with their FPKM count. (XLSX $11 \mathrm{~kb}$ )

Additional file 7: Table of P450 orthologue gene count in related plants. Comparison of tomato P450 gene along with other related plants P450 orthologue gene count. (DOCX $20 \mathrm{~kb}$ )

\section{Abbreviations}

AtP450: Arabidopsis thaliana P450; BLAST: Basic Local Alignment Search Tool; CYP: Cytochrome P450; FPKM: Fragments Per Kilobase of Transcript per Million mapped reads; MEGA: Molecular Evolutionary Genetics Analysis; P450: Cytochrome P450; RT-qPCR: Real Time Quantitative Polymerase Chain Reaction; SIP450: Solanum lycoperscium Cytochrome P450

\section{Acknowledgements}

The authors thank Dr. Angelica Lindlöf, University of Skövde, Sweden for sharing Perl script for promoter analysis. We extend words of appreciation for Dr. A. S. Kashikar, Department of Statistics, S. P. Pune University, Pune for her support and help during statistical analysis and Prof. S. S. Bhargava her for valuable suggestions. The authors thank Dr. Asaph Aharoni for providing Solanum lycoperscium L. cv MicroTom seeds and tissues specific RNA sequencing data. We are grateful to the University Grant Commission (UGC), India for financial support in the form of Start-Up grant received by VTB. The authors acknowledges Savitribai Phule Pune University for publication fee assistance.

\section{Funding}

This study was funded by the University Grant Commission (UGC), India under the scheme of Start-Up grant received by VTB.

\section{Availability of data and materials}

All the data obtained in the current study have been presented in this article.

\section{Authors' contributions}

APV carried out the analysis of the sequences, cloning, RT-qPCR and drafted the manuscript. VTB participated in its design, coordination and supervised the study. Both the authors read and approved the final manuscript.

Ethics approval and consent to participate Not applicable.

\section{Consent for publication}

Not applicable.

\section{Competing interests}

The authors declare that they have no competing interests.

\section{Publisher's Note}

Springer Nature remains neutral with regard to jurisdictional claims in published maps and institutional affiliations.

Received: 17 July 2018 Accepted: 28 January 2019

Published online: 07 February 2019

\section{References}

1. Ortiz De Montellano PR. Cytochrome P450: structure, Mechanism, and Biochemistry; 1986.

2. Schuler MA, Werck-Reichhart D. Functional genomics of P450s. Annu Rev Plant Biolagy. 2003;54:629-37.

3. Nelson DR. Cytochrome P450 and the individuality of species. Arch Biochem Biophys. 1999;369:1-10.

4. Fischer M, Knoll M, Sirim D, Wagner F, Funke S, Pleiss J. The cytochrome P450 engineering database: a navigation and prediction tool for the cytochrome P450 protein family. Bioinformatics. 2007;23:2015-7. https://doi. org/10.1093/bioinformatics/btm268. 
5. Bak S, Beisson F, Bishop G, Hamberger B, Höfer R, Paquette S, et al. Cytochromes P450. In: The Arabidopsis Book American Society Of Plant Biologist; 2011. p. e0144.

6. Gribskov M, McLachlan AD, Eisenberg D. Profile analysis: detection of distantly related proteins. Proc Natl Acad Sci U S A. 1987;84:4355-8.

7. Degtyarenko KN. Structural domains of P450-containing monooxygenase systems. Protein Eng. 1995;8:737-47.

8. Morant M, Bak S, Møller BL, Werck-Reichhart D. Plant cytochromes P450: tools for pharmacology, plant protection and phytoremediation. Curr Opin Biotecnol. 2003;14:151-62.

9. Mizutani M, Ohta D. Two isoforms of NADPH:Cytochrome P450 reductase in Arabidopsis thaliana. Gene structure, heterologous expression in insect cells, and differential regulation. Plant Physiol. 1998;116:357-67.

10. Schuler MA. Plant cytochrome P450 monooxygenases. Crit Rev Plant Sci. 1996;15:235-84

11. Baldwin WS, Marko PB, Nelson DR. The cytochrome P450 (CYP) gene superfamily in Daphnia pulex. BMC Genomics. 2009;10:169. https://doi.org/ 10.1186/1471-2164-10-169.

12. Ohnishi T, Yokota T, Mizutani M. Insights into the function and evolution of P450s in plant steroid metabolism. Phytochemistry. 2009;70:1918-29.

13. Ohmura E, Nakamura T, Tian RH, Yahara S, Yoshimitsu H, Nohara T. 26Aminocholestanol derivative, a novel key intermediate of steroidal alkaloids, from Solanum abutiloides. Tetrahedron Lett. 1995;36:8443-4.

14. Guttikonda SK, Trupti J, Bisht NC, Chen H, An Y-QC, Pandey S, et al. Whole genome co-expression analysis of soybean cytochrome $\mathrm{P} 450$ genes identifies nodulation-specific P450 monooxygenases. BMC Plant Biol. 2010; 10(1):243.

15. Ma B, Luo Y, Jia L, Qi X, Zeng Q, Xiang Z, et al. Genome-wide identification and expression analyses of cytochrome P450 genes in mulberry (Morus notabilis). J Integr Plant Biol. 2014;56:887-901.

16. Babu PR, Rao KV, Reddy VD. Structural organization and classification of cytochrome P450 genes in flax (Linum usitatissimum L.). Gene. 2013;513(1): 156-62.

17. Xie MM, Gong DP, Li FX, Liu GS, Sun YH. Genome-wide analysis of cytochrome P450 monooxygenase genes in the tobacco. Yi chuan. 2013;35: 379-87.

18. The Tomato Genome Consortium. The tomato genome sequence provides insights into fleshy fruit evolution. Nature. 2012;485:635-41.

19. Gupta S, Shi X, Lindquist IE, Devitt N, Mudge J, Rashotte AM. Transcriptome profiling of cytokinin and auxin regulation in tomato root. J Exp Bot. 2013; 64:695-704.

20. Nelson DR, Koymans L, Kamataki T, Stegeman JJ, Feyereisen R, Waxman DJ, et al. P450 superfamily: update on new sequences, gene mapping, accession numbers and nomenclature. Pharmacogenet Genomics. 1996;6:1-42.

21. Nelson DR. The cytochrome P450 homepage. Hum Genomics. 2009;4(1):59-65.

22. Edgar RC, Drive RM, Valley M. Muscle: multiple sequence alignment with high accuracy and high throughput. Nucleic Acids Res. 2004;32:1792-7.

23. Tamura K, Stecher G, Peterson D, Filipski A, Kumar S. MEGA6: molecular evolutionary genetics analysis version 6.0. Mol Biol Evol. 2013;30:2725-9.

24. Saitou N, Nei M. The neighbor joining method: a new method for reconstructing phylogenetic trees. Mol Biol Evol. 1987:4:406-25.

25. Trifinopoulos J, Nguyen LT, von Haeseler A, Minh BQ. W-IQ-TREE: a fast online phylogenetic tool for maximum likelihood analysis. Nucleic Acids Res. 2016:44:W232-5

26. Kalyaanamoorthy S, Minh BQ, Wong TKF, Von Haeseler A, Jermiin LS ModelFinder: fast model selection for accurate phylogenetic estimates. Nat Methods. 2017;14:587-9.

27. Hoang DT, Chernomor O, Von Haeseler A, Minh BQ, Vinh LS. UFBoot2: improving the ultrafast bootstrap approximation. Mol Biol Evol. 2018;35:518-22.

28. Thompson JD, Gibson TJ, Plewniak F, Jeanmougin F, Higgins DG. The CLUSTAL-X windows interface: flexible strategies for multiple sequence alignment aided by quality analysis tools. Nucleic Acids Res. 1997;25:4876-82.

29. Crooks GE, Hon G, Chandonia J-M, Brenner SE. WebLogo: a sequence logo generator. Genome Res. 2004:14:1188-90.

30. Barvkar VT, Pardeshi VC, Kale SM, Kadoo NY, Gupta VS. Phylogenomic analysis of UDP glycosyltransferase 1 multigene family in Linum usitatissimum identified genes with varied expression patterns. BMC Genomics. 2012;13(1):175.

31. Paquette SM, Bak S, Feyereisen R. Intron-exon organization and phylogeny in a large superfamily, the paralogous cytochrome P450 genes of Arabidopsis thaliana. DNA Cell Biol. 2000;19:307-17.
32. Higo K, Ugawa Y, Iwamoto M, Korenaga T. Plant cis-acting regulatory DNA elements (PLACE) database: 1999. Nucleic Acids Res. 1999;27:297-300.

33. Lescot M, Déhais $P$, Thijs $G$, Marchal $K$, Moreau $Y$, Van de Peer $Y$, et al. PlantCARE, a database of plant cis-acting regulatory elements and a portal to tools for in silico analysis of promoter sequences. Nucleic Acids Res. 2002;30:325-7.

34. Quinlan AR, Hall IM. BEDTools: a flexible suite of utilities for comparing genomic features. Bioinformatics. 2010;26:841-2.

35. Lindlöf A, Bräutigam M, Chawade A, Olsson O, Olsson B. In silico analysis of promoter regions from cold-induced genes in rice (Oryza sativa L.) and Arabidopsis thaliana reveals the importance of combinatorial control. Bioinformatics. 2009;25:1345-8.

36. Metsalu T, Vilo J. ClustVis: a web tool for visualizing clustering of multivariate data using principal component analysis and heatmap. Nucleic Acids Res. 2015:43:W566-70.

37. Chomczynski P. Single-step method of RNA isolation by acid Guanidinium extraction. Anal Biochem. 1987:159:156-9.

38. Roths MJ, Tanese N, Goff SP. Purification and characterization of murine retroviral reverse transcriptase expressed in Escherichia coli. J Biol Chem. 1985;260:9326-35.

39. Livak KJ, Schmittgen TD. Analysis of relative gene expression data using realtime quantitative PCR and the 2- $\Delta \Delta C$ Tmethod. Nat Methods. 2001;25:402-8.

40. Nelson DR. Plant cytochrome P450s from moss to poplar. Phytochem Rev. 2006:5:193-204.

41. Cramer CL, Edwards K, Dron M, Liang X, Dildine SL, Bolwell GP, et al. Phenylalanine ammonia-lyase gene organization and structure. Plant Mol Biol. 1989;12:367-83.

42. Hatton D, Sablowski R, Yung M, Smith C, Schuch W, Bevan M. Two classes of cis sequences contribute to tissue specific expression of a PAL2 promoter in transgenic tobacco. Plant J. 1995;7:859-76.

43. Bate NJ, Sivasankar S, Moxon C, Riley JMC, Thompson JE, Rothstein SJ. Molecular characterization of an Arabidopsis gene encoding hydroperoxide lyase, a cytochrome P450 that is wound inducible. Plant Physiol. 1998;117: 1393-400.

44. Alvarez-Buylla ER, Liljegren SJ, Pelaz S, Gold SE, Burgeff C, Ditta GS, et al. MADS-box gene evolution beyond flowers: expression in pollen, endosperm, guard cells, roots and trichomes. Plant J. 2000;24:457-66.

45. Ma H, Yanofsky MF, Meyerowitz EM. AGL1-AGL6, an Arabidopsis gene family with similarity to floral homeotic and transcription factor genes. Genes Dev. 1991;5:484-95.

46. Colombo L, Franken J, Koetje E, van Went J, Dons HJ, Angenent GC, et al. The petunia MADS box gene FBP11 determines ovule identity. Plant Cell. 1995:7:1859-68

47. Zhang H, Forde BG. An Arabidopsis MADS box gene that controls nutrientinduced changes in root architecture. Science. 1998:279:407-9.

48. Giuliano G, Pichersky E, Malik VS, Timko MP, Scolnik PA, Cashmore AR. An evolutionarily conserved protein binding sequence upstream of a plant light-regulated gene. Proc Natl Acad Sci. 1988;85:7089-93.

49. Ueda T, Pichersky E, Malik VS, Cashmore AR. Level of expression of the tomato rbcS-3A gene is modulated by a far upstream promoter element in a developmentally regulated manner. Plant Cell. 1989;1:217-27.

50. Donald RG, Cashmore AR. Mutation of either $\mathrm{G}$ box or I box sequences profoundly affects expression from the Arabidopsis rbcS-1A promoter. EMBO J. 1990;9:1717.

51. Tiwari $S B$, Hagen $G$, Guilfoyle $T$. The roles of auxin response factor domains in auxin-responsive transcription. The Plant Cell. 2003;15(2):533-43.

52. Morgan RW, Christman MF, Jacobson FS, Storz G, Ames BN. Hydrogen peroxide-inducible proteins in Salmonella typhimurium overlap with heat shock and other stress proteins. Proc Natl Acad Sci. 1986;83:8059-63.

53. Storozhenko S, De PP, Van MM, Inze D, Kushnir S. The heat-shock element is a functional component of the Arabidopsis APX1 gene promoter. Plant Physiol. 1998:118(3):1005-14.

54. Cubas P, Lauter N, Doebley J, Coen E. The TCP domain: a motif found in proteins regulating plant growth and development. Plant J. 1999;18:215-22

55. Li S. The Arabidopsis thaliana TCP transcription factors: a broadening horizon beyond development. Plant Signal Behav. 2015;10:e1044192.

56. Kieffer M, Master V, Waites R, Davies B. TCP14 and TCP15 affect internode length and leaf shape in Arabidopsis. Plant J. 2011;68:147-58.

57. Field B, Osbourn AE Metabolic diversification-independent assembly of operon-like gene clusters in different plants. Science. 2008;320:543-7. 
58. Ayabe S, Akashi T. Cytochrome P450s in flavonoid metabolism. Phytochem Rev. 2006;: $: 271-82$.

59. Kutchan T. Alkaloid biosynthesis: the basis for metabolic engineering of medicinal plants. Plant Cell. 1995;7:1059. https://doi.org/10.2307/3870057.

60. Nelson D, Werck-Reichhart D. A P450-centric view of plant evolution. Plant J. 2011;66:194-211.

61. Kim HB, Schaller H, Goh CH, Kwon M, Choe S, An CS, et al. Arabidopsis cyp51 mutant shows postembryonic seedling lethality associated with lack of membrane integrity. Plant Physiol. 138:2033-47.

62. Nafisi M, Goregaoker S, Botanga CJ, Glawischnig E, Olsen CE, Halkier BA, et al. Arabidopsis cytochrome P450 monooxygenase 71A13 catalyzes the conversion of indole-3-acetaldoxime in camalexin synthesis. Plant Cell. 2007; 19:2039-52.

63. Frey M, Schullehner K, Dick R, Fiesselmann A, Gierl A. Benzoxazinoid biosynthesis, a model for evolution of secondary metabolic pathways in plants. Phytochemistry. 2009;70:1645-51

64. Zhang L, Li Z, Li J, Wang A. Ectopic overexpression of SsCBF1, a CRT/DREbinding factor from the nightshade plant Solanum lycopersicoides, confers freezing and salt tolerance in transgenic Arabidopsis. PLoS One. 2013;8: e61810

65. Roy SW, Gilbert W. Rates of intron loss and gain: implications for early eukaryotic evolution. Proc Natl Acad Sci United States Am. 2005;102:5773-8.

66. Palmer JD, Logsdon JM. The recent origins of introns. Curr Opin Genet Dev. 1991;1:470-7.

67. Lan Z, Kai W, Jun TAN, Wei LI, Songgang LI. Putative cytochrome P450 genes in rice genome ( Oryza sativa L . Ssp . Indica ) and their EST evidence. Sci China Ser C Life Sci. 2002:45.

Ready to submit your research? Choose BMC and benefit from:

- fast, convenient online submission

- thorough peer review by experienced researchers in your field

- rapid publication on acceptance

- support for research data, including large and complex data types

- gold Open Access which fosters wider collaboration and increased citations

- maximum visibility for your research: over $100 \mathrm{M}$ website views per year

At $\mathrm{BMC}$, research is always in progress.

Learn more biomedcentral.com/submissions 